3. L. Markus, Generic properties of differential equations, Internat. Sympos. on Nonlinear Differential Equations and Nonlinear Mechanics, Academic Press, New York, 1963.

4. R. D. Nussbaum, Periodic solutions of analytic functional differential equations are analytic, Michigan Math. J. 20 (1973), 249-255. MR 48 \#677.

5. W. M. Oliva, Functional differential equations on compact manifolds and an approximation theorem, J. Differential Equations 5 (1969), 483-496. MR 38 \#5247.

6. M. M. Peixoto, On an approximation theorem of Kupka and Smale, J. Differential Equations 3 (1966), 214-227. MR 35 \#499.

7. S. Smale, Stable manifolds for differential equations and diffeomorphisms, Ann. Scuola Norm. Sup. Pisa (3) 17 (1963), 97-116. MR 29 \#2818b.

LEFSCHETZ CENTER FOR DYNAMICAL SYSTEMS, DIVISION OF APPLIED MATHEMATICS, BROWN UNIVERSITY, PROVIDENCE, RHODE ISLAND 02912

BULLETIN OF THE

\title{
THE SELBERG TRACE FORMULA FOR CONGRUENCE SUBGROUPS
}

\author{
BY DENNIS A. HEJHAL
}

Communicated by S. Eilenberg, March 13, 1975

1. Introduction. The Selberg trace formula for $\operatorname{SL}(2, \mathbf{R})$ is conimonly understood to be a non-Abelian analog of the Poisson summation formula. The formula arises from letting a Fuchsian group $\Gamma$ act on the upper half-plane $H$ and contains four basic contributions: identity, hyperbolic, elliptic, and parabolic [2, pp. 95-108], [3, pp. 72-79]. Because of its possible numbertheoretic applications, it seems only natural to calculate the trace formula explicitly for various congruence subgroups of $\operatorname{SL}(2, Z)$ and see what happens.

From the general theory, one knows that the parabolic (or arithmetic) contribution will be $\operatorname{Tr}(M)$, where

$$
\begin{aligned}
M= & \frac{1}{4 \pi} \int_{-\infty}^{\infty} h(r) \Phi^{\prime}(s) \Phi(s)^{-1} d r+1 / 4[I-\Phi(1 / 2)] h(0) \\
& -\left[g(0) \ln 2+\frac{1}{2 \pi} \int_{-\infty}^{\infty} h(r) \frac{\Gamma^{\prime}}{\Gamma}(1+i r) d r\right] I .
\end{aligned}
$$

AMS (MOS) subject classifications (1970). Primary 10D10, $10 \mathrm{HO5.}$ 
We use here the notation of $[2$, p. 108] and write $s=1 / 2+i r . \Phi(s)$ is the socalled Eisenstein matrix. $M$ will therefore be a $C \times C$ matrix, where $C=$ the number of inequivalent cusps. For the other three contributions, see [2, pp. 95-108].

The groups $\Gamma$ we want to consider are::SL(2,Z), $\Gamma_{0}(N), \Gamma_{1}(N), \Gamma_{2}(N)$. These last three groups are defined by the congruence conditions:

$$
\left(\begin{array}{ll}
a & b \\
c & d
\end{array}\right) \equiv\left(\begin{array}{ll}
* & * \\
0 & *
\end{array}\right), \pm\left(\begin{array}{ll}
1 & * \\
0 & 1
\end{array}\right), \pm\left(\begin{array}{ll}
1 & 0 \\
0 & 1
\end{array}\right) \bmod N
$$

We will assume that $N \geqslant 3$ is square-free. In order to evaluate the parabolic contribution for each group, one must first calculate $\Phi(s)$ and then $\operatorname{Tr}(M)$. The necessary computations are quite lengthy (for large $N$ ).

2. The trace formulas. Because we are interested only in the "arithmetic" part of the formulas, we need only give $\operatorname{Tr}(M)$. We introduce the number-theoretic tunctions $\phi(n), \Lambda(n), \omega(n)$ as in [1, pp. 233, 253, 354] and let $\epsilon= \pm 1$.

Case $\operatorname{SL}(2, \mathrm{Z}) . \quad C=1$ :

$$
\begin{aligned}
\operatorname{Tr}(M)= & g(0) \ln (\pi / 2)-\frac{1}{2 \pi} \int_{-\infty}^{\infty} h(r)\left\{\frac{\Gamma^{\prime}}{\Gamma}(1 / 2+i r)+\frac{\Gamma^{\prime}}{\Gamma}(1+i r)\right\} d r \\
& +2 \sum_{n=1}^{\infty} \frac{\Lambda(n)}{n} g(2 \ln n) .
\end{aligned}
$$

Case $\Gamma_{0}(N) . C=2^{\omega(N)}$ :

$\operatorname{Tr}(M)=C \operatorname{Tr}\left(M_{\mathrm{SL}(2, \mathrm{Z})}\right)-C g(0) \ln N-C \sum_{p \mid N} \sum_{n=p^{r}} \frac{\Lambda(n)}{n} g(2 \ln n)$.

Case $\Gamma_{1}(N) . C=1 / 2 \phi(N) 2^{\omega(N)}$ :

$$
\begin{aligned}
\operatorname{Tr}(M)= & (1 / 8) h(0) 2^{\omega(N)}[\phi(N)-2]+C g(0)[\ln (\pi / 2)-(3 / 2) \ln N] \\
& -\frac{C}{2 \pi} \int_{-\infty}^{\infty} h(r)\left\{\frac{\Gamma^{\prime}}{\Gamma}(1 / 2+i r)+\frac{\Gamma^{\prime}}{\Gamma}(1+i r)\right\} d r \\
& +C\left[2 \sum_{n \equiv \epsilon \bmod N} \frac{\Lambda(n)}{n} g(2 \ln n)\right] \\
& +2^{\omega(N)} \sum_{p \mid N} F\left(\frac{N}{p}\right)\left[\frac{1}{2} g(0) \ln p+\sum_{\substack{n \equiv \epsilon \bmod (N / p) ; \\
n=p^{r}}} \frac{\Lambda(n)}{n} g(2 \ln n)\right]
\end{aligned}
$$

where $F(k)=1 / 2 \phi(k)$ when $k \geqslant 2$, and $F(1)=1$. 
Case $\Gamma_{2}(N) . \quad C=1 / 2 \Pi_{p \mid N}\left(p^{2}-1\right)$ :

$$
\begin{aligned}
\operatorname{Tr}(M)= & (1 / 8) h(0) \prod_{p \mid N}(p+1) \cdot[\phi(N)-2]+C g(0)[\ln (\pi / 2)-2 \ln N] \\
& -\frac{C}{2 \pi} \int_{-\infty}^{\infty} h(r)\left\{\frac{\Gamma^{\prime}}{\Gamma}(1 / 2+i r)+\frac{\Gamma^{\prime}}{\Gamma}(1+i r)\right\} d r \\
+ & C\left[2 \sum_{n \equiv \epsilon \bmod N} \frac{\Delta(n)}{n} g(2 \ln n)\right] \\
+ & \prod_{p \mid N}(p+1) \cdot\left[g(0) \sum_{p \mid N} F\left(\frac{N}{p}\right) \frac{\ln p}{p+1}\right. \\
& \left.+2 \sum_{p \mid N n \equiv \epsilon \bmod (N / p) ;} \sum_{n=p^{r}} \frac{\Delta(n)}{n(p+1)} F\left(\frac{N}{p}\right) g(2 \ln n)\right] .
\end{aligned}
$$

Due to limitations of space, we will omit the formulation of the $\Phi(s)$ matrices. The case $\Gamma=\operatorname{SL}(2, Z)$ can be found in $[2, \mathrm{p} .46]$.

3. Connections with analytic number theory. Trace formulas are important for several reasons. One reason is that they exhibit a very striking structural similarity to certain explicit formulas of prime number theory (especially Weil [5]). Thus, in the notation of [2], we have

$$
\begin{aligned}
\sum_{\gamma} h(\gamma)= & h\left(\frac{i}{2}\right)+h\left(-\frac{i}{2}\right)-g(0) \ln \pi+\frac{1}{2 \pi} \int_{-\infty}^{\infty} h(r) \frac{\Gamma^{\prime}}{\Gamma}\left(\frac{1}{4}+\frac{1}{2} i r\right) d r \\
& -2 \sum_{n=1}^{\infty} \frac{\Lambda(n)}{\sqrt{n}} g(\ln n),
\end{aligned}
$$

where $1 / 2+i \gamma$ are the nontrivial zeros of $\zeta(s)$ (of course $\gamma \in \mathbf{C}$ ). One might possibly hope to interpret Weil's formula as actually being a trace formula (or else limit thereof). This would provide some important insight into the Riemann hypothesis.

If the formulas of $\$ 2$ offer any clue at all, we see that there will be several important obstructions:

(a) $2 \ln n$ and $n$ appear in place of $\ln n$ and $\sqrt{n}$;

(b) the $\Lambda(n)$ terms appear with a "+" sign instead of a "-";

(c) the identity and hyperbolic contributions to the trace formula significantly "overshadow" the parabolic (in magnitude).

4. Hecke operators. As mentioned in [3] , [4] , one can also develop a trace formula for $\operatorname{Tr}(M L)$, where $M$ is a modular correspondence and $L$ is the 
usual integral operator $L f(z)=\int_{H} k(z, w) f(w) d w$. The case of $\Gamma=\operatorname{SL}(2, \mathbf{Z})$ and $M=T_{p}$ (the usual Hecke operator) can be computed explicitly. The only serious difficulty arises from matrices $\tau=\left(\begin{array}{ll}A & B \\ C & D\end{array}\right) \in \mathrm{GL}(2, \mathrm{Z})$ having $A D-B C$ $=p$ and $|A+D|=p+1$. The corresponding contribution to $\operatorname{Tr}\left(T_{p} L\right)$ is

$$
\begin{aligned}
g(\ln p)\left[p^{1 / 2} \ln p+2 p^{1 / 2} \ln \pi+2 p^{1 / 2} \ln \left(p^{1 / 2}-p^{1 / 2}\right)\right]-\frac{2 g(\ln p)}{p^{1 / 2}-p^{-1 / 2}} \sum_{k=1}^{p-1} \ln (k, p-1) \\
-1 / 2 p^{1 / 2} h(0)+2 p^{1 / 2} \sum_{n=1}^{\infty} \frac{\Lambda(n)}{n}[g(2 \ln n-\ln p)+g(2 \ln n+\ln p)] \\
+p^{1 / 2} \int_{\ln p}^{\infty} g(u) \frac{e^{u}+1}{\left(e^{u / 2}+p^{1 / 2}\right)\left(e^{u / 2}-p^{-1 / 2}\right)} d u \\
-\frac{1}{2 \pi} \int_{-\infty}^{\infty} h(r)\left[p^{1 / 2+i r}+p^{1 / 2-i r}\right] \frac{\Gamma^{\prime}}{\Gamma}(1 / 2+i r) d r .
\end{aligned}
$$

One finds here a certain resemblance to the formulas of $\S 2$. As a result, the obstructions (a)-(c) still seem to apply.

5. Detailed proofs will be published elsewhere.

\section{REFERENCES}

1. G. H. Hardy and E. M. Wright, An introduction to the theory of numbers, 4th Ed., Oxford Univ. Press, London, 1960.

2. T. Kubota, Elementary theory of Eisenstein series, Halsted Press, New York, 1973.

3. A. Selberg, Harmonic analysis and discontinuous groups in weakly symmetric Riemannian spaces with applications to Dirichlet series, J. Indian Math. Soc. 20 (1956), 47-87. MR 19, 531.

4. - Discontinuous groups and harmonic analysis, Proc. Internat. Congress Math. (Stockholm, 1962), Inst. Mittag-Leffler, Djursholm, 1963, pp. 177-189. MR 31 \#372.

5. A. Weil, Sur les "formules explicites" de la théorie des nombres premiers, Comm. Sém. Math. Univ. Lund (Medd. Lunds Univ. Mat. Sem.), Tome supplémentaire, 1952, 252-265. MR 14, 727.

\section{NEW YORK 10027 \\ DEPARTMENT OF MATHEMATICS, COLUMBIA UNIVERSITY, NEW YORK,}

